\title{
25 Research Soure \\ Relationship Between Tooth Loss and Sarcopenia in Chinese Suburban Community-Dwelling Older Adults
}

\section{Feng Wang}

Shanghai University of Traditional Chinese Medicine Jingru Wang

Shanghai University of Traditional Chinese Medicine

Peipei Han

Shanghai University of Medicine and Health Sciences

Yuewen Liu

Shanghai University of Medicine and Health Sciences

\section{Weibo Ma}

Shanghai University of Traditional Chinese Medicine

\section{Hui Zhang}

Shanghai University of Traditional Chinese Medicine

\section{Ning Wu}

Shanghai University of Medicine and Health Sciences

\section{Sijia Sang}

Shanghai University of Medicine and Health Sciences

\section{Yining Xia}

Shanghai University of Medicine and Health Sciences

\section{Jiangtao Pan}

Shanghai University of Medicine and Health Sciences

\section{Yang Liu}

Shanghai University of Medicine and Health Sciences

\section{Fandi Xie}

Shanghai Hongkou District Jiangwan Hospital

\section{Shumeng Niu}

Shanghai Hongkou District Jiangwan Hospital

\section{$\mathrm{HaO} \mathrm{Hu}$}

Shanghai Hongkou District Jiangwan Hospital

\section{Hongbing Wang}

Shanghai Fourth Rehabilitation Hospital

Ying Yu 
Shanghai University of Traditional Chinese Medicine

Qi Guo ( $\square$ guoqijp@gmail.com )

Shanghai University of Medicine and Health Sciences Affiliated Zhoupu Hospital

\section{Research Article}

Keywords: tooth loss, grip strength, walking speed, sarcopenia

Posted Date: July 28th, 2021

DOl: https://doi.org/10.21203/rs.3.rs-528293/v2

License: (c) (1) This work is licensed under a Creative Commons Attribution 4.0 International License. Read Full License 


\section{Abstract}

Background: Both sarcopenia and loss of teeth are associated with aging. The purpose of this study was to investigate potential relationships between tooth loss and sarcopenia and components of sarcopenia, including declining muscle mass, muscle strength, and physical performance, in Chinese suburban community-dwelling older adults.

Methods: The subjects were 1494 people over 60 years of age (average age: $71.64 \pm 5.97$ years $₫ \mathrm{men}$, $\mathrm{n}=609$ ) from Chongming District of Shanghai and Hangu District of Tianjin. The Asian Working Group for Sarcopenia (AWGS) criteria were used to define sarcopenia. The three basic diagnostic components of sarcopenia (muscle mass, muscle strength, and physical performance) were assessed using a bioelectrical impedance analyzer, a grip strength test, and a four-meter walk test, respectively. The subjects were divided into groups depending on self-reported loss of teeth.

Results: After adjusting for confounding variables, we found no correlation between tooth loss and sarcopenia or muscle mass. However, the walking speed of female participants with at least 10 teeth lost was $0.059 \mathrm{~m} / \mathrm{s}$ slower than that of participants with fewer than 10 teeth lost (Pख0.001), and grip strength was $1.577 \mathrm{~kg}$ lower among male participants with at least 10 teeth lost than among males with fewer than 10 teeth lost $(P=0.023)$.

Conclusion: Our results suggest that tooth loss is negatively correlated with muscle strength in males and average walking speed in females. These results are consistent with the importance of good oral hygiene in preventing declines of physical performance in older adults.

\section{Main Text}

China's aging problem is becoming increasingly serious, with 164.5 million people aged 65 and above ${ }^{[1]}$. At the same time, incidence of age-related sarcopenia is also on the rise, and it has become an important focus of geriatric research. Sarcopenia is defined as the age-related loss of indicators of skeletal muscle quality, including muscle mass, strength and physical performance. In older adults, sarcopenia is associated with an increase in adverse outcomes, including falls, functional decline, weakness and death $^{[2,3]}$; therefore, active prevention and treatment of sarcopenia is particularly important.

The older adults also face increasingly serious problems with oral health associated with declines of physical and mental health. Oral health problems can have a significant impact on the elderly, leading to discomfort, pain and tooth loss. Tooth loss, in particular, is an indicator of poor oral health ${ }^{[4]}$, and the number of missing teeth may reflect the cumulative level of oral inflammation ${ }^{[5]}$. In addition, the local inflammation caused by tooth loss can induce a systemic inflammatory response ${ }^{[6]}$. These systemic changes, such as changes in serum levels of the inflammatory biomarkers C-reactive protein and interleukin, also occur in muscle injury. The inflammation associated with poor oral health may, then, affect physical fitness, especially muscle mass, muscle strength and muscle function ${ }^{[7]}$. In addition, adult 
tooth loss may affect physical fitness and contribute to sarcopenia by reducing diet quality and the intake of nutrients ${ }^{[8]}$. Therefore, it is reasonable to think that tooth loss is causally related to sarcopenia and its components.

In a recent study, poor oral health, including tooth loss, was shown to be a predictor of future sarcopenia in community-dwelling older adults ${ }^{[9]}$, while two other studies identified tooth loss as a risk factor for sarcopenia in adults ${ }^{[10,11]}$. Despite the significant correlations that have been identified, there are relatively few studies on tooth loss and sarcopenia, and those that have been performed typically did not evaluate denture status, which has been shown to impact nutritional status ${ }^{[12]}$. Therefore, it is necessary to establish whether tooth loss is a risk factor for sarcopenia in the elderly. In addition, the relationship between tooth loss and muscle mass, strength and function, which are indicators of sarcopenia, remains controversial $^{[13-18]}$. There are also few comprehensive studies on tooth loss relative to the specific components of sarcopenia, muscle mass, muscle strength, and physical performance.

Here, we conducted a cross-sectional study to determine the relationship between tooth loss and sarcopenia and its contributing factors among Chinese suburban community-dwelling older adults, so as to provide a reference for predicting decreased physical performance and identifying high-risk groups. Given the ease with which oral health interventions can be integrated into daily life, it is important to determine whether such interventions can be used to reduce age-related risks of sarcopenia and decline of physical performance.

\section{Materials And Methods}

Participants. Our study population included residents from Hangu District, Tianjin and Chongming District, Shanghai, China. Participants were selected from 1614 individuals of age 60 years or above in these areas who joined the national free physical examination program conducted from August 2018 and June to July 2019. Participants with the following conditions were excluded from the study: (1) inability to communicate with interviewers or to grant informed consent; (2) inability to perform the handgrip strength test or the $4 \mathrm{~m}$ walking test; (3) failure to complete body composition measurement; or (4) failure to complete the questionnaire about oral care. The final study population comprised 1494 subjects. This research was approved by the Ethics Committee at Tianjin Medical University and Shanghai University of Medicine and Health Sciences, and informed consent was obtained from all patients.

Assessment of the number of teeth. Participants arriving at community health service centers submitted a questionnaire via a face-to-face interview. Tooth status was assessed via a self-reported measure that asked participants about number of teeth lost. Possible answers were none, 1, 2, from 3 to 5 , from 6 to 9 , and 10 or more. Participants were then categorized into two groups based on the number of teeth missing: from 0 to 9 teeth lost or 10 or more teeth lost. Participants also self-reported denture status.

Assessment of sarcopenia. We used the definition from the Asian Working Group for Sarcopenia to assess sarcopenia ${ }^{[19]}$. Here, a person with low muscle mass, low muscle strength and/or low physical 
performance was identified as having sarcopenia. Low muscle mass was defined as a relative skeletal muscle mass index, which is the ratio of appendicular skeletal muscle mass (ASM) to height $(\mathrm{Ht})^{2}$, of less than $7.0 \mathrm{~kg} / \mathrm{m}^{2}$ in men and $5.7 \mathrm{~kg} / \mathrm{m}^{2}$ in women. Low physical performance was defined as an average walking speed of less than or equal to $0.8 \mathrm{~m} / \mathrm{s}$. Low muscle strength was defined as a grip pressure of less than $26 \mathrm{~kg}$ for men and less than $18 \mathrm{~kg}$ for women.ASM values were obtained using a bioelectrical impedance analyzer (Inbody720; Bio space Co., Ltd, Seoul, Korea).

Assessment of muscle strength. Muscle strength was assessed via average grip strength of two attempts at maximum effort with a participant's dominant hand. Grip strength was measured using a dynamometer (Grip-D; Takei Ltd, Niigata, Japan).

Assessment of physical performance. Walking speed was used as a marker of physical performance. To measure walking speed, we placed two photocells connected to a recording timer at the beginning and end of a $4 \mathrm{~m}$ course on site. Participants were asked to stand with their feet touching the starting line. When the tester gave the "start" command, participants began to walk at a normal speed, and the time between the activation of the first and second photocells was recorded.

Assessment of covariates. Sociodemographic and behavioral characteristics and medical conditions were obtained from the participants through face-to-face consultations and questionnaires.

Sociodemographic variables, including age, gender, marital status and educational level were assessed. Weight and height were measured, and body mass index $\left(\mathrm{kg} / \mathrm{m}^{2}\right)$ was calculated. Marital status was categorized as widowed or not. Living conditions were divided into living alone or not. The level of education was divided into literate and illiterate. Behavioral characteristics included information on smoking (never, former smoker, or current smoker) and drinking (never, former drinker, occasional or daily drinker) habits and were obtained from the questionnaire. Family annual income was also included, and categories were less than 1000 yuan, 1000-3000 yuan, 3000 to 5000 yuan, and greater than 5000 yuan. The presence of specific medical conditions, including diabetes, hypertension, dyslipidemia, were established using standardized criteria that combined information from history of physical illness evaluated on the basis of participants' responses to questions, physician diagnoses, and statuses of corresponding treatments, pharmacological or otherwise. Denture status was divided into two groups: those with partial dentures and full dentures, and those without dentures. The short form of the International Physical Activity Questionnaire (IPAQ) was used to evaluate physical activity ${ }^{[20]}$. The Geriatric Depression Scale (GDS) was used to assess depressive symptoms in older adults; a GDS score of at least 11 was defined as depression ${ }^{[21]}$. We assessed nutritional status with Mini Nutritional Assessment (MNA) scores ${ }^{[22]}$.

Statistical analyses. We separated the participants by sex and classified the number of teeth lost into two groups ( 0 to 9 and at least 10). Analysis of variance (ANOVA) and chi-square tests were conducted to compare the characteristics and lifestyle habits of individuals between the two groups. Binary logistic regression models and multivariate linear regression models were used for modelling the relationships among the three basic diagnostic components of sarcopenia and the number of teeth lost. Model 1 
controlled for the covariates age and BMI; Model 2 was based on model 1, with additional adjustments of IPAQ results, income, literacy, smoking, drinking and denture status; Model 3 was based on model 2, with additional adjustments of depression, nutrition status, hypertension and diabetes. Bilateral $P<0.05$ was considered to be statistically significant. All statistical analyses were performed using IBM SPSS Statistics 25.0 .

\section{Results}

The data of 1614 participants from Hangu District, Tianjin and Chongming District, Shanghai were collected in August 2018 and June to July 2019. A total of 1494 participants were included for analysis in this study. Table 1 and Table 2 show the socioeconomic characteristics, demographics and disease statuses of the participants. Of the participants, 609 (40.7\%) were male. It was found that age, BMI, relative skeletal muscle mass index, grip strength, average walking speed, overall sarcopenia status, and denture status were significantly different between the groups that were categorized according to the number of teeth lost (Table 1 and Table 2).

A binary logistic regression model was applied, and the data were adjusted for the covariates age, BMI, district of residency, IPAQ results, income, literacy, smoking and drinking practice, denture status, depression, nutrition score, hypertension, and diabetes. We did not identify a statistical relationship between tooth loss and sarcopenia (Table 3). Next, a multivariate linear regression model was developed, after adjusting for the potentially confounding factors. In this analysis, muscle mass was not associated with tooth loss in either sex group. In men, grip strength was $1.577 \mathrm{~kg}$ lower in those with at least 10 teeth lost as compared with those with nine or fewer teeth lost $(P=0.023)$. Among women, participants who had lost at least 10 teeth had an average walking speed that was $0.059 \mathrm{~m} / \mathrm{s}$ slower than that of participants with nine or fewer teeth lost $(P<0.001)$ (Table 4).

\section{Discussion}

Our research examined associations between self-reported tooth loss and sarcopenia and its components (muscle mass, muscle strength, and physical performance) in Chinese suburban community-dwelling older adults after adjusting for various confounders. Considering that the association between the number of teeth and physical performance remains controversial, the results of this representative study are important.

\section{The relationship between tooth loss and sarcopenia}

Our study disagrees with previous research that has linked general sarcopenia to oral health. In one recent study, subjects with fewer than 20 natural teeth had an adjusted odds ratio for sarcopenia of 1.96 for men and 2.86 for women, compared with those with full dentition ${ }^{[10]}$. Another cross-sectional study also showed that impaired dentition was significantly associated with sarcopenia in Japanese adults living in the community ${ }^{[11]}$. In a meta-analysis of 27 studies reviewed, a possible association between 
oral health status and sarcopenia was found; however, most of the studies included in this analysis were cross-sectional studies in Japan, and the results therefore are not representative ${ }^{[23]}$.

On the other hand, after adjusting for covariate factors, we found no association between tooth loss and sarcopenia in either sex. One possible explanation for the disagreement may result from the varying ages of study participants. Age is an important factor in sarcopenia: physical function declines by approximately $4 \%$ every year after the age of 65 , and movement disorders become more common, affecting $24 \%$ of adults over 75 years old ${ }^{[24,25]}$. We used relatively young older adults, and the age range of the population included in our study fairly small; male and female study participants were aged, on average, $71.94 \pm 5.97$ years and $71.44 \pm 5.97$ years, respectively. In other studies, either the ages of participants exceeded 75 years or the range of ages of inclusion was too wide to permit generalizable claims ${ }^{[10,11]}$. These differences in participant ages, then, may explain why other groups found relationships between tooth loss and sarcopenia, even though no such relationships seem to exist in agematched groups. Notably, thus far, associations between tooth loss and sarcopenia have been reported only rarely, and no firm conclusions have been drawn. Further large longitudinal studies are needed to test these proposed relationships.

\section{The relationship between tooth loss and muscle mass}

While connections between sarcopenia in general and tooth loss are controversial, more detailed studies have suggested that links between tooth loss and one component of sarcopenia, low muscle mass, may occur through inflammatory and nutritional pathways ${ }^{[26,27]}$. For example, one key factor in tooth loss, periodontitis, increases levels of interleukin- 6 and tumor necrosis factor-alpha in gingival tissue.

Resultant systemic increases of these inflammatory cytokines can cause loss of muscle mass ${ }^{[28,29]}$. In support of this mechanistic connection, Akinari et al found that tooth loss was an independent risk factor for decreased muscle mass in men but not in women ${ }^{[18]}$. Notably, there are limitations to this study, especially regarding the relatively small sample size.

In a recent longitudinal study, poor oral health, including tooth loss, was shown to be a predictor of future muscle loss in older people living in the community ${ }^{[9]}$. A Korean study similarly showed that participants with at least 20 remaining natural teeth had more muscle mass on average than those with fewer than 20 remaining natural teeth. While this latter study ostensibly demonstrated a connection in men and women, after correcting for confounding factors, the number of remaining teeth and muscle mass were found to correlate in men but not in women over age $65^{[30]}$. It is speculated that the reason for gender differences in this correlation may be that men and women tend to make different food choices, with women tending to eat more fruits and vegetables than men. This lifestyle connection may lead to a limiting of protein intake in women and thus gender-related differences in muscle mass dynamics.

Our findings are inconsistent with several previous studies, however. Although we did identify differences in muscle mass between men and women, after adjusting for confounders, we found no correlation between tooth loss and muscle mass. One reason for these differences may involve the assessment tool. 
We used a reliable method that is specified by Asian Working Group for Sarcopenia ${ }^{[19]}$, and this method would be expected to lead to highly accurate results. In addition, our study population lives in the suburban community, and many of the participants were of low economic status and had been engaged in agricultural labor during their working years. These physical factors could have influenced muscle development and the impact of inflammatory factors arising from gingivitis.

\section{The relationship between tooth loss and grip strength}

Grip strength is a commonly used indicator of total body strength and is a known predictor of disability and mortality ${ }^{[31]}$. A recent Chinese study showed no link between tooth loss and grip strength in people over the age of $60^{[32]}$. In a Japanese community study involving participants ages 40 to 70 , there was also no correlation found between the number of teeth and grip strength in either sex ${ }^{[18]}$. The results of this latter study are limited because of the lack of consideration of confounding factors, including socioeconomic status and the use of dentures, both of which have clear connections to tooth loss. In particular, the use of dentures can improve the chewing ability of older adults and thus can improve nutrient intake and nutritional status, which would affect physical performance and muscle strength ${ }^{[33]}$.

On the other hand, in a South Korean study, while associations between the number of teeth and grip strength disappeared entirely in women after adjusting for socioeconomic status, use of dentures, health behavioral factors and general health, statistically significant connections remained in men ${ }^{[14]}$. Hamalainen et al also found a positive correlation between the number of teeth and grip strength in men over the age of 80 , but found no correlation in women ${ }^{[17]}$. A recent study similarly reported an association between tooth loss and mortality in older men, but not in women ${ }^{[34]}$. These findings are consistent with the results of our present study.

The differences in associations between men and women are difficult to explain. Variabilities in socioeconomic factors and social activity may be important influences on differences between men and women, and these factors may explain the lack of association between tooth loss and grip strength in women. In addition, reported results are not directly comparable, since there were differences in age distribution, methods of measuring grip strength, and adjustment for confounding factors in the previous studies $^{[14,17,18,32]}$. Therefore, gender differences in the relationship between the number of teeth and grip strength is intriguing and deserves further study.

\section{The relationship between tooth loss and walking speed}

Walking speed is a clinically useful indicator of mobility and overall health status in older adults and an independent predictor of adverse outcomes such as disability and mortality ${ }^{[35,36]}$. Decreases in walking speed likely result from a variety of factors that occur throughout life. Previous studies have confirmed that tooth loss is associated with slower average walking speed ${ }^{[37]}$. In addition, circulation levels of biomarkers of inflammation have been shown to be negatively associated with walking speed $^{[38]}$. The mechanism of association can be explained in that an elevated level of proinflammatory 
cytokines in the serum of patients with periodontal disease may change the metabolism within local muscles groups leading to a decline in physical performance ${ }^{[39]}$. Accordingly, a recent cohort study showed that tooth loss was associated with an accelerated decline in walking speed in older adults, inflammation may play a role in the association between tooth loss and walking speed

decline ${ }^{[40]}$. However, the population included in this study had a higher education, and the results may not be applicable to older suburban-dwelling adults with lower literacy rates.

Our study found that even after adjustment for confounding variables, tooth loss was associated with walking speed in women, but not in men. Our results are similar to a study from Japan ${ }^{[18]}$. Men, therefore, seem to be less prone to age-related bone and muscle decline than are postmenopausal women. While sex steroids can stimulate peak bone and muscle mass growth in adolescence and middle age, and prevent subsequent loss in older men, they have no effect on postmenopausal women ${ }^{[41]}$. Women are particularly vulnerable to decreased walking speed because of the dramatic decline in estrogen levels after menopause ${ }^{[42]}$. Men, conversely, tend to have enough muscle in their lower limbs to overcome the high rate of decrease of muscle mass with age ${ }^{[18]}$. These factors may explain our result that tooth loss was significantly related to walking speed in women, but not in men. Preventing tooth loss, then, may be more important for women.

\section{Strength and Limitations}

Some limitations of this study are worth mentioning. First, measurement of oral health status was based on self-reports, not clinical examination. However, the validity and reliability of self-reported oral health has been established by multiple studies, and this method is widely used in epidemiological surveys. Secondly, this study is a cross-sectional study and is not a study of causal relationships between tooth loss and sarcopenia and its components; causal relationships should be addressed in future longitudinal studies. Finally, some older adults with poor physical performance may have been excluded from the study because it was not convenient for them to leave home to participate in a physical examination. The participants in this study are therefore older adults with good activity levels, and this self-selection may have an impact on the research results.

This study also has many strengths. First, for older adults, reducing tooth loss can improve functioning and reduce risks associated with physical decline. This result informs us that caregivers or individuals can prevent or reduce physical performance decline by improving oral health. Second, the study provided additional data regarding the oral health of a suburban community-dwelling population, and it therefore fills an important gap in understanding.

\section{Conclusion}

Our research found that tooth loss is not related to sarcopenia and muscle mass, but tooth loss is related to physical performance and muscle strength. These associations provide the possibility of improving the 
physical function of the older adults by improving oral health. The factors identified in this study can be used in suburban community health plans to maintain the physical function of older adults.

\section{Declarations}

Ethics approval. This study was approved by the Shanghai University of Medicine and Health Sciences ethics committee. All participants signed an informed consent form to participate before data collection. We confirm that all methods were performed in accordance with the relevant guidelines and regulations.

\section{Acknowledgements}

The authors thank all the staff and participants who participated in this study.

\section{Authors Contributions}

Feng Wang and Jingru Wang were major contributor in writing the manuscript and contributed to the statistical analysis. Peipei Han and Yuewen Liu contributed to guide the statistical analysis. Weibo Ma, Hui Zhang, Ning Wu, Sijia Sang, Yining Xia, Jiangtao Pan, Fandi Xie, Shumeng Niu, Hao Hu, Hongbing Wang and Yang Liu contributed to the conception or design of the work and data collection. Ying Yu and Qi Guo guided the other authors in data analysis. Qi Guo directed the revision of the manuscript. All authors read and approved the final manuscript.

\section{Funding}

This work was supported by Shanghai Sailing Program (20YF1418200), Scientific Research Foundation of SUMHS『SSF-21-03-005『and Wu Jieping medical foundation (320.6750.17073).

\section{Additional information}

Competing Interests The authors declare that they have no competing interests.

\section{References}

[1] Fang EF, Xie C, Schenkel JA, et al. A research agenda for ageing in china in the 21st century (2nd edition): focusing on basic and translational research, long-term care, policy and social networks[J]. AGEING RES REV. 2020®101174.

[2] Cruz-Jentoft AJ, Sayer AA. Sarcopenia[J]. LANCET. 2019,393(10191)『2636-2646.

[3] Dodds RM, Roberts HC, Cooper C, et al. The epidemiology of sarcopenia[J]. J CLIN DENSITOM. $2015,18(4) \varangle 461-466$. 
[4] Liljestrand JM, Havulinna AS, Paju S, et al. Missing teeth predict incident cardiovascular events, diabetes, and death[J]. J DENT RES. 2015,94(8)ه1055-1062.

[5] Kassebaum NJ, Bernabe E, Dahiya M, et al. Global burden of severe tooth loss: a systematic review and meta-analysis[J]. J DENT RES. 2014,93(7 Suppl)®20S-28S.

[6] Nelwan SC, Nugraha RA, Endaryanto A, et al. Converging findings from linkage between periodontal pathogen with atopic and allergic immune response[J]. CYTOKINE. 2019,113囚89-98.

[7] Bramantoro T, Hariyani N, Setyowati D, et al. The impact of oral health on physical fitness: a systematic review[J]. Heliyon. 2020,6(4)囚e3774.

[8] Iwasaki M, Taylor GW, Manz MC, et al. Oral health status: relationship to nutrient and food intake among 80-year-old japanese adults[J]. Community Dent Oral Epidemiol. 2014,42(5) $₫ 441-450$.

[9] Tanaka T, Takahashi $\mathrm{K}$, Hirano $\mathrm{H}$, et al. Oral frailty as a risk factor for physical frailty and mortality in community-dwelling elderly[J]. J Gerontol A Biol Sci Med Sci. 2018,73(12)凶1661-1667.

[10] Han $\mathrm{CH}$, Chung JH. Association between sarcopenia and tooth loss[J]. Ann Geriatr Med Res. 2018,22(3) $₫ 145-150$.

[11] Iwasaki M, Kimura $\mathrm{Y}$, Ogawa $\mathrm{H}$, et al. The association between dentition status and sarcopenia in japanese adults aged $>/=75$ years[J]. J ORAL REHABIL. 2017,44(1)ष51-58.

[12] Gupta A, Khandelwal R, Kapil U. Interrelationship between dental health status and nutritional status among elderly subjects in india[J]. J Family Med Prim Care. 2019,8(2)ه477-481.

[13] Eremenko M, Pink C, Biffar R, et al. Cross-sectional association between physical strength, obesity, periodontitis and number of teeth in a general population[J]. J CLIN PERIODONTOL. 2016,43(5) \401-407.

[14] Shin HS. Handgrip strength and the number of teeth among korean population[J]. J PERIODONTOL. 2019,90(1)ष90-97.

[15] Welmer AK, Rizzuto D, Parker MG, et al. Impact of tooth loss on walking speed decline over time in older adults: a population-based cohort study[J]. AGING CLIN EXP RES. 2017,29(4)®793-800.

[16] Ramsay SE, Papachristou E, Watt RG, et al. Influence of poor oral health on physical frailty: a population-based cohort study of older british men[J]. J AM GERIATR SOC. 2018,66(3) $₫ 473-479$.

[17] Hamalainen $P$, Rantanen $T$, Keskinen $M$, et al. Oral health status and change in handgrip strength over a 5-year period in 80-year-old people[J]. GERODONTOLOGY. 2004,21(3)ه155-160.

[18] Inui A, Takahashi I, Sawada K, et al. Teeth and physical fitness in a community-dwelling 40 to 79 year-old japanese population[J]. CLIN INTERV AGING. 2016,11区873-878. 
[19] Chen LK, Liu LK, Woo J, et al. Sarcopenia in asia: consensus report of the asian working group for sarcopenia[J]. J AM MED DIR ASSOC. 2014,15(2)ष95-101.

[20] Craig CL, Marshall AL, Sjostrom M, et al. International physical activity questionnaire: 12-country reliability and validity[J]. Med Sci Sports Exerc. 2003,35(8) $₫ 1381-1395$.

[21] Yesavage JA, Brink TL, Rose TL, et al. Development and validation of a geriatric depression screening scale: a preliminary report[J]. J PSYCHIATR RES. 1982,17(1)®37-49.

[22] Vellas B, Guigoz Y, Garry PJ, et al. The mini nutritional assessment (mna) and its use in grading the nutritional state of elderly patients[J]. NUTRITION. 1999,15(2)ه116-122.

[23] Hatta K, Ikebe K. Association between oral health and sarcopenia: a literature review[J]. J PROSTHODONT RES. 2020.

[24] Milanovic Z, Pantelic S, Trajkovic N, et al. Age-related decrease in physical activity and functional fitness among elderly men and women[J]. CLIN INTERV AGING. 2013,8®549-556.

[25] Fried LP, Ferrucci L, Darer J, et al. Untangling the concepts of disability, frailty, and comorbidity: implications for improved targeting and care[J]. J Gerontol A Biol Sci Med Sci. 2004,59(3)囚255-263.

[26] El-Shinnawi U, Soory M. Associations between periodontitis and systemic inflammatory diseases: response to treatment[J]. Recent Pat Endocr Metab Immune Drug Discov. 2013,7(3) $₫ 169-188$.

[27] Ritchie CS, Joshipura K, Hung HC, et al. Nutrition as a mediator in the relation between oral and systemic disease: associations between specific measures of adult oral health and nutrition outcomes[J]. Crit Rev Oral Biol Med. 2002,13(3)ष291-300.

[28] Visser M, Pahor M, Taaffe DR, et al. Relationship of interleukin- 6 and tumor necrosis factor-alpha with muscle mass and muscle strength in elderly men and women: the health abc study[J]. J Gerontol A Biol Sci Med Sci. 2002,57(5)『M326-M332.

[29] Loos BG, Craandijk J, Hoek FJ, et al. Elevation of systemic markers related to cardiovascular diseases in the peripheral blood of periodontitis patients[J]. J PERIODONTOL. 2000,71(10)ه1528-1534.

[30] Kim S, Min JY, Lee HS, et al. The association between the number of natural remaining teeth and appendicular skeletal muscle mass in korean older adults[J]. Ann Geriatr Med Res. 2018,22(4)ه194-199.

[31] Rantanen T, Volpato S, Ferrucci L, et al. Handgrip strength and cause-specific and total mortality in older disabled women: exploring the mechanism[J]. J AM GERIATR SOC. 2003,51(5)囚636-641.

[32] Zhou Z, Gu Y, Zhang Q, et al. Association between tooth loss and handgrip strength in a general adult population[J]. PLOS ONE. 2020,15(7)囚e236010. 
[33] Moriya S, Tei K, Murata A, et al. Influence of dental treatment on physical performance in communitydwelling elderly persons[J]. GERODONTOLOGY. 2012,29(2)『e793-e800.

[34] Morita I, Nakagaki H, Kato K, et al. Relationship between survival rates and numbers of natural teeth in an elderly japanese population[J]. GERODONTOLOGY. 2006,23(4)®214-218.

[35] Studenski S, Perera S, Patel K, et al. Gait speed and survival in older adults[J]. JAMA. 2011,305(1)凶5058.

[36] Guralnik JM, Ferrucci L, Pieper CF, et al. Lower extremity function and subsequent disability: consistency across studies, predictive models, and value of gait speed alone compared with the short physical performance battery[J]. J Gerontol A Biol Sci Med Sci. 2000,55(4)『M221-M231.

[37] Brand C, Bridenbaugh SA, Perkovac M, et al. The effect of tooth loss on gait stability of communitydwelling older adults[J]. GERODONTOLOGY. 2015,32(4)『296-301.

[38] Gardner AW, Montgomery PS, Casanegra Al, et al. Association between gait characteristics and endothelial oxidative stress and inflammation in patients with symptomatic peripheral artery disease[J]. Age (Dordr). 2016,38(3) \64.

[39] Hoppe CB, Oliveira J, Grecca FS, et al. Association between chronic oral inflammatory burden and physical fitness in males: a cross-sectional observational study[J]. INT ENDOD J. 2017,50(8)『740-749.

[40] Welmer AK, Rizzuto D, Parker MG, et al. Impact of tooth loss on walking speed decline over time in older adults: a population-based cohort study[J]. AGING CLIN EXP RES. 2017,29(4)囚793-800.

[41] Laurent MR, Dedeyne L, Dupont J, et al. Age-related bone loss and sarcopenia in men[J]. MATURITAS. 2019,122区51-56.

[42] Kuo LC, Polson AM, Kang T. Associations between periodontal diseases and systemic diseases: a review of the inter-relationships and interactions with diabetes, respiratory diseases, cardiovascular diseases and osteoporosis[J]. PUBLIC HEALTH. 2008,122(4)ه417-433.

\section{Tables}




\begin{tabular}{|c|c|c|c|}
\hline \multirow[t]{3}{*}{ Variable } & \multicolumn{2}{|c|}{ Number of tooth loss } & \multirow[t]{3}{*}{$\mathrm{p}$} \\
\hline & 0 to 9 & $\geq 10$ & \\
\hline & $(n=335)$ & $(n=274)$ & \\
\hline $\operatorname{Age}(y)$ & $70.07 \pm 5.26$ & $74.23 \pm 6.00$ & $₫ 0.001$ \\
\hline BMI & $24.25 \pm 3.03$ & $22.93 \pm 3.54$ & $\varangle 0.001$ \\
\hline $\mathrm{ASM} / \mathrm{Ht}^{2}\left(\mathrm{~kg} / \mathrm{m}^{2}\right)$ & $7.86 \pm 0.97$ & $7.51 \pm 0.79$ & $₫ 0.001$ \\
\hline Grip strength (kg) & $31.13 \pm 7.44$ & $27.57 \pm 7.78$ & $₫ 0.001$ \\
\hline Walk speed (m/s) & $1.14 \pm 0.24$ & $1.07 \pm 0.25$ & 0.001 \\
\hline IPAQ (Met/week) & $4164(1533,8400)$ & $3786(1626,6426)$ & 0.033 \\
\hline Widowed, n (\%) & $25(7.5)$ & $33(12.0)$ & 0.057 \\
\hline Living alone, n (\%) & $36(10.7)$ & $33(12.0)$ & 0.615 \\
\hline Illiteracy & $22(6.6)$ & $27(9.9)$ & 0.251 \\
\hline Income冈n (\%) & & & 0.363 \\
\hline$\otimes 1000$ & 49(14.7) & $40(14.7)$ & \\
\hline Smoking, n (\%) & & & $₫ 0.001$ \\
\hline Current smokers\ & $92(27.5)$ & $111(40.7)$ & \\
\hline Drinking, n (\%) & & & 0.003 \\
\hline Daily drinkers & $78(23.6)$ & $78(29.0)$ & \\
\hline Nutritional status & & & 0.014 \\
\hline well-nourished & $272(81.2)$ & 195(71.2) & \\
\hline at risk of malnutrition & 61(18.2) & $76(27.7)$ & \\
\hline malnourished & $2(0.6)$ & $3(1.1)$ & \\
\hline \multicolumn{4}{|l|}{ Disease『n (\%) } \\
\hline Hypertension & $223(66.6)$ & $171(62.4)$ & 0.285 \\
\hline hypercholesterolemia & $204(60.9)$ & $139(50.7)$ & 0.012 \\
\hline Diabetes & $54(16.1)$ & 53(19.3) & 0.298 \\
\hline Stoke & $26(7.8)$ & $22(8.1)$ & 0.897 \\
\hline Sarcopenia & 19(5.7) & $40(14.6)$ & $\otimes 0.001$ \\
\hline
\end{tabular}




\begin{tabular}{|c|c|c|c|}
\hline Depression & $32(9.8)$ & $27(9.9)$ & 0.954 \\
\hline Cognitive impairment & $38(11.8)$ & $40(15.2)$ & 0.241 \\
\hline \multicolumn{3}{|l|}{ Brushing frequency, $\mathrm{n}(\%)$} & 0.161 \\
\hline$\geq 2$ time /day & 113(33.7) & $78(28.4)$ & \\
\hline$\otimes 2$ time /day & $222(66.3)$ & 196(71.6) & \\
\hline \multicolumn{3}{|l|}{ Brushing time, $\mathrm{n}(\%)$} & 0.616 \\
\hline$\triangle 2 \mathrm{~min}$ & $181(53.9)$ & $153(56.0)$ & \\
\hline$\geq 2 \min$ & $154(46.1)$ & $121(44.0)$ & \\
\hline Gingival bleeding, n (\%) & $44(13.2)$ & $14(5.2)$ & 0.001 \\
\hline Denture status, n (\%) & $86(25.7)$ & $184(67.2)$ & $\mathbb{\nabla 0 . 0 0 1}$ \\
\hline \multicolumn{4}{|c|}{ Note: Data are shown as mean \pm SD or number (percentage). } \\
\hline \multicolumn{4}{|c|}{$\begin{array}{l}\text { Abbreviation: BMI, body mass index; ASM/Ht2, ASM, appendicular lean mass; } \mathrm{Ht} \text {, Height; .IPAQ, } \\
\text { International Physical Activity Questionnaire; MET/week, metabolic equivalent task minutes per week; }\end{array}$} \\
\hline
\end{tabular}




\begin{tabular}{|c|c|c|c|}
\hline \multirow[t]{3}{*}{ Variable } & \multicolumn{2}{|c|}{ Number of tooth loss } & \multirow[t]{3}{*}{$\mathrm{p}$} \\
\hline & 0 to 9 & $\geq 10$ & \\
\hline & $(n=436)$ & $(n=449)$ & \\
\hline Age(y) & $69.42 \pm 5.19$ & $73.40 \pm 6.03$ & $\otimes 0.001$ \\
\hline BMI & $24.73 \pm 3.62$ & $23.93 \pm 3.47$ & 0.001 \\
\hline $\mathrm{ASM} / \mathrm{Ht}^{2}\left(\mathrm{~kg} / \mathrm{m}^{2}\right)$ & $6.36 \pm 0.94$ & $6.08 \pm 0.79$ & $\varangle 0.001$ \\
\hline Grip strength $(\mathrm{kg})$ & $19.09 \pm 5.54$ & $17.46 \pm 5.56$ & $\varangle 0.001$ \\
\hline Walk speed (m/s) & $1.08 \pm 0.17$ & $0.99 \pm 0.23$ & $\varangle 0.001$ \\
\hline IPAQ (Met/week) & $4519(2373,8363)$ & $4110(1688,8078)$ & 0.643 \\
\hline Widowed, n (\%) & $91(20.9)$ & 142(31.6) & 0.001 \\
\hline Living alone, n (\%) & $60(13.8)$ & $101(22.5)$ & 0.001 \\
\hline Illiteracy & $106(24.4)$ & $110(24.5)$ & 0.691 \\
\hline Income冈n (\%) & & & 0.956 \\
\hline$\otimes 1000$ & $93(21.3)$ & $97(21.7)$ & \\
\hline Smoking, n (\%) & & & 0.433 \\
\hline Current smokers\ & $27(6.2)$ & $37(8.3)$ & \\
\hline Drinking, n (\%) & & & 0.104 \\
\hline Daily drinkers & $13(3.1)$ & $24(5.4)$ & \\
\hline Nutritional status & & & 0.288 \\
\hline well-nourished & $344(78.8)$ & $340(75.8)$ & \\
\hline at risk of malnutrition & $91(21.0)$ & 105(23.3) & \\
\hline malnourished & $1(0.2)$ & $4(0.9)$ & \\
\hline \multicolumn{4}{|l|}{ Disease囚n (\%) } \\
\hline Hypertension & $279(64.0)$ & $316(70.4)$ & 0.043 \\
\hline hypercholesterolemia & $302(69.3)$ & $319(71.0)$ & 0.563 \\
\hline Diabetes & $90(20.7)$ & 84(18.8) & 0.479 \\
\hline Stoke & $20(4.7)$ & $25(5.6)$ & 0.528 \\
\hline Sarcopenia & $43(9.9)$ & $80(17.8)$ & 0.001 \\
\hline
\end{tabular}




\begin{tabular}{|c|c|c|c|}
\hline Depression & $54(12.6)$ & $57(12.9)$ & 0.881 \\
\hline Cognitive impairment & 79(19.3) & $103(24.5)$ & 0.067 \\
\hline \multicolumn{3}{|l|}{ Brushing frequency, $\mathrm{n}(\%)$} & 0.862 \\
\hline$\geq 2$ time / day & 192(44.0) & $200(44.6)$ & \\
\hline$\bigotimes 2$ time /day & $244(56.0)$ & $249(55.4)$ & \\
\hline \multicolumn{3}{|l|}{ Brushing time, $\mathrm{n}(\%)$} & 0.255 \\
\hline$\otimes 2 \mathrm{~min}$ & $268(61.5)$ & $259(57.7)$ & \\
\hline$\geq 2 \min$ & 168(38.5) & $190(42.3)$ & \\
\hline Gingival bleeding, n (\%) & $70(19.6)$ & $45(10.5)$ & $\varangle 0.001$ \\
\hline Denture status, n (\%) & 154(35.3) & $333(74.2)$ & $₫ 0.001$ \\
\hline \multicolumn{4}{|c|}{ Note: Data are shown as mean \pm SD or number (percentage). } \\
\hline \multicolumn{4}{|c|}{$\begin{array}{l}\text { Abbreviation: BMI, body mass index; } \mathrm{ASM} / \mathrm{Ht} 2 \text {, ASM, appendicular lean mass; } \mathrm{Ht} \text {, Height; .IPAQ, } \\
\text { International Physical Activity Questionnaire; MET/week, metabolic equivalent task minutes per week, }\end{array}$} \\
\hline
\end{tabular}

Table 3.Binary logistic regression of sarcopenia, showing effects as odds ratios and $95 \%$ confidence intervals

\begin{tabular}{|c|c|c|c|c|c|c|}
\hline \multirow{2}{*}{$\begin{array}{c}\text { Independent } \\
\text { variable }\end{array}$} & \multicolumn{2}{|l|}{ Model 1} & \multicolumn{2}{|l|}{ Model 2} & \multicolumn{2}{|l|}{ Model 3} \\
\hline & $\mathrm{OR}(95 \% \mathrm{CI})$ & p-value & $\mathrm{OR}(95 \% \mathrm{CI})$ & p-value & OR $(95 \% \mathrm{CI})$ & $\mathrm{p}$-value \\
\hline \multicolumn{7}{|l|}{ Male } \\
\hline 0 to 9 & Ref. & & Ref. & & Ref. & \\
\hline$\geq 10$ & $1.369(0.703-2.665)$ & 0.356 & $1.633(0.751-3.547)$ & 0.216 & $1.585(0.696-3.231)$ & 0.251 \\
\hline \multicolumn{7}{|l|}{ Female } \\
\hline 0 to 9 & Ref. & & Ref. & & Ref. & \\
\hline$\geq 10$ & $1.368(0.843-2.218)$ & 0.205 & $1.242(0.727-2.123)$ & 0.428 & $1.261(0.727-2.188)$ & 0.409 \\
\hline
\end{tabular}

Abbreviation: OR: odds ratio; CI: confidence interval.

Note: Model 1: adjusted for Age, BMI, District. Model 2: adjusted for Age, BMI, District, IPAQ, Income, Illiteracy, Smoking, Drinking, Denture status. Model 3: adjusted for Age, BMI, District, IPAQ, Income, Illiteracy, Smoking, Drinking, Denture status, Depression, Nutrition status, Hypertension, Diabetes.

Table 4. Linear association between the number of tooth loss and $\mathrm{ASM} / \mathrm{Ht}^{2} \square \mathrm{Grip}$ strength几Walk speed 


\begin{tabular}{|c|c|c|c|c|c|c|}
\hline \multirow{2}{*}{ Independent variable } & \multicolumn{2}{|c|}{ Model 1} & \multicolumn{2}{|c|}{ Model 2} & \multicolumn{2}{|c|}{ Model 3} \\
\hline & $\beta$ & $\mathrm{p}$-value & $\beta$ & $\mathrm{p}$-value & $\beta$ & $\mathrm{p}$-value \\
\hline \multicolumn{7}{|l|}{$\operatorname{ASM}(\mathrm{HH})^{2}\left(\mathrm{~kg} / \mathrm{m}^{2}\right)$} \\
\hline \multicolumn{7}{|l|}{ Male } \\
\hline 0 to 9 & Ref. & & Ref. & & Ref. & \\
\hline$\geq 10$ & -0.066 & 0.289 & -0.100 & 0.150 & -0.083 & 0.212 \\
\hline \multicolumn{7}{|l|}{ Female } \\
\hline 0 to 9 & Ref. & & Ref. & & Ref. & \\
\hline$\geq 10$ & -0.044 & 0.364 & -0.011 & 0.830 & -0.013 & 0.810 \\
\hline \multicolumn{7}{|l|}{ Grip strength (kg) } \\
\hline \multicolumn{7}{|l|}{ Male } \\
\hline 0 to 9 & Ref. & & Ref. & & Ref. & \\
\hline$\geq 10$ & -1.254 & 0.043 & -1.707 & 0.013 & -1.577 & 0.023 \\
\hline \multicolumn{7}{|l|}{ Female } \\
\hline 0 to 9 & Ref. & & Ref. & & Ref. & \\
\hline$\geq 10$ & -0.614 & 0.112 & 0.797 & 0.055 & -0.730 & 0.083 \\
\hline \multicolumn{7}{|l|}{ Walk speed $(\mathrm{m} / \mathrm{s})$} \\
\hline \multicolumn{7}{|l|}{ Male } \\
\hline 0 to 9 & Ref. & & Ref. & & Ref. & \\
\hline$\geq 10$ & -0.029 & 0.149 & -0.041 & 0.063 & -0.036 & 0.107 \\
\hline \multicolumn{7}{|l|}{ Female } \\
\hline 0 to 9 & Ref. & & Ref. & & Ref. & \\
\hline$\geq 10$ & -0.044 & 0.001 & -0.063 & $<0.001$ & -0.059 & $<0.001$ \\
\hline
\end{tabular}

Note: Model 1: adjusted for Age, BMI, District. Model 2: adjusted for Age, BMI, District, IPAQ, Income, Illiteracy, Smoking, Drinking, Denture status. Model 3: adjusted for Age, BMI, District, IPAQ, Income, Illiteracy, Smoking, Drinking, Denture status, Depression, Nutrition status, Hypertension, Diabetes. 